\title{
Uniqueness results for the Dirichlet problem for higher order elliptic equations in polyhedral angles
}

\author{
Sara Monsurrò ${ }^{1 * \dagger}$, llia Tavkhelidze ${ }^{2 \dagger}$ and Maria Transirico ${ }^{1 \dagger}$
}

\section{"Correspondence:}

smonsurro@unisa.it

1 Dipartimento di Matematica,

Università di Salerno, Via Giovann

Paolo II, 132, Fisciano, 84084, Italy

†Equal contributors

Full list of author information is

available at the end of the article

\begin{abstract}
We consider the Dirichlet boundary value problem for higher order elliptic equations in divergence form with discontinuous coefficients in polyhedral angles. Some uniqueness results are proved.

MSC: 35J30; 35J40
\end{abstract}

Keywords: higher order elliptic equations; Dirichlet problem; discontinuous coefficients

\section{Introduction}

The Dirichlet problem for the polyharmonic equation in a bounded domain of $\mathbb{R}^{n}$ has been studied by Sobolev in [1]. Later on, different problems (Dirichlet, Neumann and Riquier problems) for harmonic, biharmonic and meta-harmonic functions have been considered by Vekua in [2] and [3], both in the cases of bounded and unbounded domains of $\mathbb{R}^{n}$. Successively, many authors studied analogous problems in more general cases and with different methods (see, for instance, [4-10], the general survey on this subject [11] and the references quoted therein). In particular, in [10], the author obtains the uniqueness of the solution of the Dirichlet problem,

$$
\begin{cases}\Delta^{m} u(x)=0 & \text { in } \mathbb{R}_{l}^{n}, \\ \left.\frac{\partial j u(x)}{\partial \vec{v}^{j}}\right|_{\partial \mathbb{R}_{l}^{n}=0,} \quad j=0, \ldots, m-1,\end{cases}
$$

where $\Delta^{m}$ denotes the polyharmonic operator of order $m, \Delta$ is the Laplace operator and $\mathbb{R}_{l}^{n}$ is a polyhedral angle of $\mathbb{R}^{n}$, defined in Section 2 . We explicitly observe that for $l=0$ the above mentioned definition gives the half-space $\mathbb{R}_{+}^{n}$. We note that, due to the tools used in the proof, some restrictions on the dimension $n$ of the space are required.

Our aim, in this paper, is to generalize the uniqueness result of [10]. More precisely, we are concerned with the following Dirichlet problem for a homogeneous equation in divergence form of order $2 m$ :

$$
\begin{cases}\sum_{|\alpha|=|\beta|=m} D^{\alpha}\left(a_{\alpha \beta}(x) D^{\beta} u(x)\right)=0 & \text { in } \mathbb{R}_{l}^{n}, \\ \left.\frac{\partial^{j} u(x)}{\partial \vec{v} j}\right|_{\partial \mathbb{R}_{l}^{n}=0,} & j=0, \ldots, m-1,\end{cases}
$$


where the discontinuous coefficients $a_{\alpha \beta}$ are bounded and measurable functions satisfying the uniform ellipticity condition.

Let us remark that if we take $\alpha=\beta$ and if the coefficients of the equation are constants $a_{\alpha \beta}(x)=\frac{m !}{\alpha !}$, then the left-hand side of the equation in (1.2) is exactly the polyharmonic operator $\Delta^{m}$ in (1.1).

Our main results consist in two uniqueness theorems obtained for some particular cases of problem (1.2). More precisely, in Section 4 we consider problem (1.2) in the case $m=1$ and in Section 5 we assume that $\alpha=\beta$. The main tool in our analysis is a generalization of the Hardy inequality proved by Kondrat'ev and Oleinik in [5] (see Section 3).

\section{Notation}

Throughout this work we use the following notation:

- $n \in \mathbb{N}$ is the dimension of the considered space;

- Greek letters denote $n$-dimensional multi-indices, for instance $\alpha=\left(\alpha_{1}, \alpha_{2}, \ldots, \alpha_{n}\right)$, where $\alpha_{i} \in \mathbb{N} \cup\{0\}, i=1, \ldots, n$;

- $|\alpha|=\alpha_{1}+\cdots+\alpha_{n}$ is the module of the multi-index $\alpha$;

- $\alpha !=\alpha_{1} ! \cdots \alpha_{n}$ ! is the factorial of the multi-index $\alpha$;

- $\varphi_{, i}(x)=\frac{\partial \varphi(x)}{\partial x_{i}}, i=1, \ldots, n$;

- $D_{i}^{\alpha_{i}}=\frac{\partial^{\alpha_{i}}}{\left(\partial x_{i}\right)^{\alpha_{i}}}, i=1, \ldots, n$

- $D^{\alpha}=D_{1}^{\alpha_{1}} \cdots D_{n}^{\alpha_{n}}$

- for $\xi=\left(\xi_{1}, \ldots, \xi_{n}\right) \in \mathbb{R}^{n}$ we set $\xi^{\alpha}=\xi_{1}^{\alpha_{1}} \ldots \xi_{n}^{\alpha_{n}}$;

- for every $l \in\{0, \ldots, n-1\}$,

$$
\mathbb{R}_{l}^{n}=\left\{x=\left(x_{1}, x_{2}, \ldots, x_{n}\right) \in \mathbb{R}^{n}: x_{i}>0, i=n-l, \ldots, n\right\}
$$

is the 'polyhedral angle' with vertex in the origin;

- for $l=0$ the above definition gives the half-space $\mathbb{R}_{+}^{n}$;

- for $\rho>0$ we denote by $Q_{\rho}=\left\{x \in \mathbb{R}_{l}^{n}:|x|<\rho\right\}$.

\section{Setting of the problem}

We want to consider the following differential equation in divergence form of order $2 m$, $m \in \mathbb{N}$, in certain unbounded domains of $\mathbb{R}^{n}, n>2$ :

$$
\sum_{|\alpha|=|\beta|=m} D^{\beta}\left(a_{\alpha \beta}(x) D^{\alpha} u(x)\right)=f(x)
$$

where $f(x)$ is a given datum and the coefficients $a_{\alpha \beta}(x)$ are bounded measurable functions satisfying the uniform ellipticity condition, i.e. there exist two positive constants $\lambda_{1}$ and $\lambda_{2}$ such that for each nonzero vector $\xi \in \mathbb{R}^{n}$ one has

$$
\lambda_{1}|\xi|^{2 m} \leq \sum_{|\alpha|=|\beta|=m} a_{\alpha \beta}(x) \xi^{\alpha} \xi^{\beta} \leq \lambda_{2}|\xi|^{2 m} \text { a.e. }
$$

Let us mention that if we take $\alpha=\beta$ in (3.1) and if the coefficients of the equation are constants $a_{\alpha}(x)=\frac{m !}{\alpha !}$, then left-hand side of this equation is the polyharmonic operator $\Delta^{m}$, where $\Delta$ denotes, as usual, the Laplace operator. 
For every sufficiently differentiable functions $u$ and $v$ let us set

$$
\begin{aligned}
& E_{m}^{a}(u, v)=\sum_{|\alpha|=|\beta|=m} a_{\alpha \beta}(x) D^{\alpha} u D^{\beta} v, \\
& E_{m}(u, v)=\sum_{|\alpha|=m} \frac{m !}{\alpha !} D^{\alpha} u D^{\alpha} v, \\
& E_{m}^{a}(u)=E_{m}^{a}(u, u), \\
& E_{m}(u)=E_{m}(u, u) .
\end{aligned}
$$

Definition 3.1 We say that the function $u$ is a generalized solution of (3.1) in $\mathbb{R}_{l}^{n}$ with homogeneous Dirichlet boundary conditions if $u \in W^{m, 2}\left(\mathbb{R}_{l}^{n}\right)$ and it satisfies the integral identity

$$
(-1)^{m} \int_{Q_{\rho}} E_{m}^{a}(u, v) d x=\int_{Q_{\rho}} f(x) v(x) d x
$$

for any $\rho>0$ and any function $v \in W_{0}^{m, 2}\left(Q_{\rho}\right)$, where $f \in L^{2}\left(\mathbb{R}_{l}^{n}\right)$.

To prove our main results, consisting in two uniqueness theorems, we will essentially use the following generalized Hardy inequality.

Lemma 3.2 (Generalized Hardy inequality) Let $p>1, j$, and $n$ be such that $j+n-p \neq 0$. Assume that for a sufficiently smooth function $g$ the following condition is fulfilled in a cone $V \subset \mathbb{R}^{n}$ with vertex in the origin of coordinates:

$$
\int_{V}|x|^{j}|\nabla g(x)|^{p} d x<\infty
$$

where $\nabla g=\left(\frac{\partial g}{\partial x_{1}}, \ldots, \frac{\partial g}{\partial x_{n}}\right)$ is the gradient of the function $g$. Then there exist two constants $M, K>0$ such that

$$
\int_{V}|x|^{j-p}|g(x)-M|^{p} d x<K \int_{V}|x|^{j}|\nabla g(x)|^{p} d x
$$

where the constant $K$ does not depend on the function $g$. If, in addition, $g(0)=0$ then $M=0$.

Remark 3.3 The previous lemma, which was proved by Kondrat'ev and Oleinik in [5], holds also if we replace (3.5) with the following inequality:

$$
\int_{V_{R_{2}} \backslash V_{R_{1}}}|x|^{j-p}|g(x)-M|^{p} d x<K \int_{V_{R_{2} \backslash V_{R_{1}}}}|x|^{j}|\nabla g(x)|^{p} d x,
$$

with $0<R_{1}<R_{2}$, where $V_{R}, R>0$, denotes the intersection between the cone $V$ and the open ball of center in the origin and radius $R$.

This result can be deduced by the proof of Lemma 3.2, with slight modifications. We point out that in this proof it is also well rendered that the constant $K$ does not depend on $R_{1}$ and $R_{2}$. 
Remark 3.4 As evidenced in many works about different variants of Hardy or CaffarelliKohn-Nirenberg type inequalities (see for instance [5,12-16]), there are always very important restrictions on the dimension of the space $n$, the order of 'singularity' $j$ and the order of the integral norm $p$.

\section{Dirichlet problem for second order elliptic equations}

In this section we consider, for $m=1$, the homogeneous equation (3.1) in the polyhedral angle $\mathbb{R}_{l}^{n}, 0 \leq l \leq n-1$, with the homogeneous Dirichlet boundary condition, namely

$$
\left\{\begin{array}{l}
\sum_{i, j=1}^{n} \frac{\partial}{\partial x_{j}}\left(a_{i j}(x) \frac{\partial u}{\partial x_{i}}\right)=0 \text { in } \mathbb{R}_{l}^{n}, \\
\left.u\right|_{\partial \mathbb{R}_{l}^{n}=0 .}
\end{array}\right.
$$

Let us observe that by Definition 3.1 it follows that every generalized solution is such that

$$
\int_{\mathbb{R}_{l}^{n}} E_{1}(u) d x<\infty
$$

Now we prove our first uniqueness result.

Theorem 4.1 Let $n>2$. Assume that (3.2) is satisfied, with $m=1$. If $u$ is a generalized solution of problem (4.1), then $u \equiv 0$ in $\mathbb{R}_{l}^{n}$.

Proof Let $\Theta(s)$ be an auxiliary function in $C_{0}^{\infty}([0, \infty[)$ defined by

$$
\Theta(s) \equiv \begin{cases}1 & 0 \leq s \leq 1 \\ \theta(s) & 1 \leq s \leq 2 \\ 0 & s \geq 2\end{cases}
$$

with $0 \leq \theta(s) \leq 1$, and such that there exists a positive constant $K_{0}$ such that

$$
\left|\Theta^{\prime}(s)\right|^{2} \leq K_{0} \Theta(s) .
$$

We note that in order to obtain a cut-off function $\Theta(s)$ of the above mentioned type one can consider a classical mollifier and modify it suitably near to $s=1$ and $s=2$.

Set, for any $R>0$,

$$
\Theta_{R}(x)=\Theta\left(\frac{|x|}{R}\right) .
$$

Note that the function $\Theta_{R}$ is such that, for any $j=1, \ldots, n$, one has

$$
\Theta_{R, j}(x)=\Theta^{\prime}\left(\frac{|x|}{R}\right) \cdot \frac{x_{j}}{R|x|} .
$$

Let us now consider the function

$$
v(x)=u(x) \cdot \Theta_{R}(x) .
$$


Clearly, by definition of $\Theta_{R}$ and as a consequence of our boundary condition, one has $v(x) \in W_{0}^{1,2}\left(Q_{2 R}\right)$. Thus, substituting this function in the integral identity (3.3), we get

$$
\int_{Q_{2 R}} \sum_{i, j=1}^{n} a_{i j}(x) u_{, i} u_{, j} \Theta_{R}(x) d x+\int_{Q_{2 R} \backslash Q_{R}} \sum_{i, j=1}^{n} a_{i j}(x) u_{, i} u \Theta_{R, j}(x) d x=0 .
$$

Hence, by (3.2) we have

$$
\int_{Q_{2 R}} E_{1}^{a}(u) \cdot \Theta_{R}(x) d x=\left|\int_{Q_{2 R} \backslash Q_{R}} \sum_{i, j=1}^{n} a_{i j}(x) u_{, i} u \Theta^{\prime}\left(\frac{|x|}{R}\right) \cdot \frac{x_{j}}{R|x|} d x\right| .
$$

Recalling that for any $\varepsilon>0$ one has

$$
a b \leq \frac{\varepsilon \cdot a^{2}}{2}+\frac{b^{2}}{2 \varepsilon}, \quad a, b \geq 0,
$$

in view of the boundedness of the coefficients and of (4.4), one gets

$$
\begin{aligned}
& \int_{Q_{2 R}} E_{1}^{a}(u) \cdot \Theta_{R}(x) d x \\
& \quad \leq \frac{\varepsilon}{2} K_{1} K_{0} \int_{Q_{2 R} \backslash Q_{R}} E_{1}(u) \cdot \Theta_{R} d x+\frac{K_{1}}{2 \varepsilon} \int_{Q_{2 R} \backslash Q_{R}} \frac{u^{2}}{|x|^{2}} d x,
\end{aligned}
$$

where $K_{0}$ is the constant in (4.4) and $K_{1}=K_{1}\left(n, \lambda_{2}\right)$. Thus, taking into account the ellipticity of the coefficients, we obtain

$$
\begin{aligned}
& \int_{Q_{2 R}} E_{1}(u) \cdot \Theta_{R}(x) d x \\
& \quad \leq \frac{\varepsilon}{2} K_{2} K_{0} \int_{Q_{2 R} \backslash Q_{R}} E_{1}(u) \cdot \Theta_{R} d x+\frac{K_{2}}{2 \varepsilon} \int_{Q_{2 R} \backslash Q_{R}} \frac{u^{2}}{|x|^{2}} d x,
\end{aligned}
$$

with $K_{2}=K_{2}\left(n, \lambda_{1}, \lambda_{2}\right)$.

Therefore, if we choose $\varepsilon=\frac{1}{K_{2} K_{0}}$ and we apply the generalized Hardy inequality (3.6) (with $p=2$ and $j=0$ ) to the second term in the right-hand side of (4.8), we deduce that

$$
\int_{Q_{2 R}} E_{1}(u) \cdot \Theta_{R}(x) d x \leq K_{3} \int_{Q_{2 R} \backslash Q_{R}} E_{1}(u) d x
$$

where the constant $K_{3}$ does not depend on the radius $R$ and on the function $u$ (see Remark 3.3).

Now, observe that clearly for any $P>0$ there exists $R>P$ such that $Q_{P} \subset Q_{R}$ and therefore, in view of the definition of $\Theta_{R}$, by the former inequality we obtain

$$
\int_{Q_{P}} E_{1}(u) d x \leq K_{3} \int_{Q_{2 R} \backslash Q_{R}} E_{1}(u) d x
$$


Condition (4.2) being satisfied, the right-hand side of (4.9) tends to zero when $R \rightarrow \infty$. Now, since the left-hand side of (4.9) is independent of the radius $R$, we have, for any $P>0$,

$$
\int_{Q_{P}} E_{1}(u) d x=\int_{Q_{P}} \sum_{i=1}^{n} u_{, i} u, i d x=0 .
$$

This means that the function $u(x)$ is a constant and, according to the boundary condition in (4.1), this constant is zero. This concludes our proof.

Remark 4.2 Note that our proof do not provide any uniqueness result for $n=2$, since in this case the generalized Hardy inequality in Lemma 3.2 does not apply, as a consequence of our choice of $p$ and $j$.

\section{Dirichlet problem for higher order elliptic equations}

Here, we consider the following homogeneous equation of order $2 m$ with homogeneous Dirichlet boundary conditions in the polyhedral angle $\mathbb{R}_{l}^{n}, l \in\{0, \ldots, n-1\}$ :

$$
\begin{cases}\sum_{|\alpha|=m} D^{\alpha}\left(a_{\alpha}(x) D^{\alpha} u(x)\right)=0 & \text { in } \mathbb{R}_{l}^{n}, \\ \left.\frac{\partial j u(x)}{\partial \vec{v} j}\right|_{\partial \mathbb{R}_{l}^{n}=0,} & j=0, \ldots, m-1 .\end{cases}
$$

Note that, again, in view of Definition 3.1 one finds that every generalized solution of problem (5.1) is such that

$$
\int_{\mathbb{R}_{l}^{n}} E_{m}(u) d x<\infty
$$

Theorem 5.1 Let $n>2 m$ or $n=2 k+1$, with $k \in \mathbb{N}$. Assume that (3.2) is satisfied. If $u$ is a generalized solution of problem (5.1), then $u(x) \equiv 0$ in $\mathbb{R}_{l}^{n}$.

Proof Let us use again the function $\Theta_{R}$ introduced in the proof of Theorem 4.1.

It is easy to check that

$$
D^{\alpha} \Theta_{R}(x)=\sum_{i=1}^{|\alpha|} \Theta^{(i)}\left(\frac{|x|}{R}\right) \cdot \frac{P_{|\alpha|}(x)}{R^{i}|x|^{(2|\alpha|-i)}},
$$

where $\Theta^{(i)}$ denotes the derivative of order $i$ of the function $\Theta$ and $P_{|\alpha|}(x)$ is a polynomial of order $|\alpha|$.

Moreover, if we assume that there exist some positive constants $K_{i}$, such that

$$
\left|\Theta^{(i)}(s)\right|^{2} \leq K_{i} \Theta(s), \quad i=1, \ldots,|\alpha|,
$$

then, for $R<|x|<2 R$, one has

$$
\left|D^{\alpha} \Theta_{R}(x)\right|^{2} \leq \frac{K_{\alpha} \Theta_{R}(x)}{|x|^{2|\alpha|}}
$$

where the constant $K_{\alpha}$ depends only on $\alpha$. 
Note that function $v(x)=u(x) \cdot \Theta_{R}(x) \in W_{0}^{m, 2}\left(Q_{2 R}\right)$, thus, substituting it in the integral identity (3.3), we deduce

$$
\begin{aligned}
\int_{Q_{2 R}} E_{m}^{a}\left(u, u \Theta_{R}\right) d x= & \int_{Q_{2 R}} E_{m}^{a}(u) \Theta_{R} d x \\
& +\int_{Q_{2 R} \backslash Q_{R}} \sum_{|\alpha|=m} a_{\alpha}(x) D^{\alpha} u\left[\sum_{\gamma+\iota=\alpha, \iota \neq 0} \frac{(|\gamma|+|\iota|) !}{\gamma ! \iota !} D^{\gamma} u D^{\iota} \Theta_{R}\right] d x=0 .
\end{aligned}
$$

Therefore

$$
\int_{Q_{2 R}} E_{m}^{a}(u) \Theta_{R} d x=\left|\int_{Q_{2 R} \backslash Q_{R}} \sum_{|\alpha|=m} a_{\alpha}(x) D^{\alpha} u\left[\sum_{\gamma+\iota=\alpha, \iota \neq 0} \frac{(|\gamma|+|\iota|) !}{\gamma ! \iota !} D^{\gamma} u D^{\iota} \Theta_{R}\right] d x\right| .
$$

From (4.7) and (5.3), arguing as in the proof of Theorem 4.1, we deduce that

$$
\begin{aligned}
\int_{Q_{2 R}} E_{m}(u) \Theta_{R}(x) d x \leq & \sum_{|\alpha|=m} K_{\alpha}^{\prime} \varepsilon_{\alpha} \int_{Q_{2 R} \backslash Q_{R}} E_{m}(u) \Theta_{R}(x) d x \\
& +\sum_{|\alpha|=m} \frac{K_{\alpha}^{\prime \prime}}{\varepsilon_{\alpha}} \int_{Q_{2 R} \backslash Q_{R}}\left[\sum_{|\gamma|=0}^{m-1} E_{|\gamma|}(u) \cdot \frac{1}{|x|^{2(m-|\gamma|)}}\right] d x,
\end{aligned}
$$

with $K_{\alpha}^{\prime}=K_{\alpha}^{\prime}\left(n, \alpha, \lambda_{1}, \lambda_{2}, K_{\alpha}\right)$ and $K_{\alpha}^{\prime \prime}=K_{\alpha}^{\prime \prime}\left(n, \alpha, \lambda_{1}, \lambda_{2}\right)$.

Now, we apply repeatedly the Hardy inequality (3.6) to the single summands of the second term in the right-hand side of (5.4) until the order of the partial derivatives achieves $m$. Thus, after an appropriate selection of $\varepsilon_{\alpha}$, we get

$$
\int_{Q_{2 R}} E_{m}(u) \Theta_{R}(x) d x \leq \widetilde{K} \int_{Q_{2 R} \backslash Q_{R}} E_{m}(u) d x,
$$

where the constant $\widetilde{K}$ is independent of the radius $R$ and of the function $u$.

Finally, following the same argument used in Theorem 4.1, for any $P>0$ we find $R>P$ such that $Q_{P} \subset Q_{R}$ and therefore, taking into account the definition of $\Theta_{R}$, we obtain

$$
\int_{Q_{P}} E_{m}(u) d x \leq \widetilde{K} \int_{Q_{2 R} \backslash Q_{R}} E_{m}(u) d x .
$$

In view of condition (5.2), the right-hand side of (5.5) goes to zero when $R \longrightarrow \infty$, and, since the left-hand side of (5.5) is independent of $R$, we have, for any $P>0$,

$$
\int_{Q_{P}} E_{m}(u) d x=\int_{Q_{P}}\left[\sum_{|\alpha|=m} \frac{m !}{\alpha !} D^{\alpha} u D^{\alpha} u\right] d x=0
$$

Therefore, the partial derivatives of any order of the solution are equal to zero, thus, as a consequence of the boundary conditions in (5.1), we deduce that $u(x) \equiv 0$ in $\mathbb{R}_{l}^{n}$.

Remark 5.2 Clearly also in this case the repeated application of the Hardy inequality yields the restrictions $n>2 m$ or $n=2 k+1, k \in \mathbb{N}$, on the space dimension. 


\section{Competing interests}

The authors declare that they have no competing interests.

\section{Authors' contributions}

The authors conceived and wrote this article in collaboration and with same responsibility. All of them read and approved the final manuscript.

\section{Author details}

${ }^{1}$ Dipartimento di Matematica, Università di Salerno, Via Giovanni Paolo II, 132, Fisciano, 84084, Italy. ${ }^{2}$ Iv. Javakhishvili Tbilisi State University, University Street 2, Tbilisi, 0186, Georgia.

\section{Authors' information}

Sara Monsurrò and Maria Transirico are members of the Gruppo Nazionale per I'Analisi Matematica, la Probabilità e le loro Applicazioni (GNAMPA) of the Istituto Nazionale di Alta Matematica (INdAM).

Received: 23 June 2014 Accepted: 8 October 2014 Published online: 04 November 2014

\section{References}

1. Sobolev, SL: Some Applications of Functional Analysis in Mathematical Physics. Izdat. Leningrad. Gos. Univ., Leningrad (1950). Translations of Mathematical Monographs, vol. 7. Am. Math. Soc., Providence (1963)

2. Vekua, IN: On metaharmonic functions. Lect. Notes TICMI 14, 62 pp. (2013). Translated from the 1943 Russian original Trav. Inst. Math. Tbilissi 12, 105-174 (1943)

3. Vekua, IN: New Methods for Solving Elliptic Equations. OGIZ, Moscow (1948) (in Russian)

4. Cassisa, C, Ricci, P, Tavkhelidze, I: Analogue of Saint-Venant's principle for the one special type 4-th order elliptic equation and its applications. Appl. Math. Inform. 4, 11-29 (1999)

5. Kondrat'ev, VA, Oleinik, OA: Boundary value problems for the system of elasticity theory in unbounded domains. Korn's inequalities. Usp. Mat. Nauk 43(5), 55-98 (1988) (in Russian)

6. Kozlov, V, Maz'ya, V: Asymptotics of a singular solution to the Dirichlet problem for an elliptic equation with discontinuous coefficients near the boundary. In: Function Spaces, Differential Operators and Nonlinear Analysis (Teistungen, 2001), pp. 75-115. Birkhäuser, Basel (2003)

7. Monsurrò, S, Transirico, M: A $L^{p}$-estimate for weak solutions of elliptic equations. Abstr. Appl. Anal. 2012, Article ID $376179(2012)$

8. Monsurrò, S, Transirico, M: Dirichlet problem for divergence form elliptic equations with discontinuous coefficients. Bound. Value Probl. 2012, Article ID 67 (2012)

9. Monsurrò, $\mathrm{S}$, Transirico, M: A priori bounds in $L^{p}$ for solutions of elliptic equations in divergence form. Bull. Sci. Math $137,851-866(2013)$

10. Tavkhelidze, I: On some properties of solutions of polyharmonic equation in polyhedral angles. Georgian Math. J. 14, 565-580 (2007)

11. Gazzola, F, Grunau, H, Sweers, G: Polyharmonic Boundary Value Problems. Positivity Preserving and Nonlinear Higher Order Elliptic Equations in Bounded Domains. Lecture Notes in Mathematics, vol. 1991. Springer, Berlin (2010)

12. Bojarski, B, Hajlasz, P: Pointwise inequalities for Sobolev functions and some applications. Stud. Math. 106, 77-92 (1993)

13. Brezis, H, Marcus, M: Hardy's inequalities revisited. Dedicated to Ennio De Giorgi. Ann. Sc. Norm. Super. Pisa, Cl. Sci. 25, 217-237 (1997)

14. Catrina, F, Wang, ZQ: On the Caffarelli-Kohn-Nirenberg inequalities: sharp constants, existence (and nonexistence), and symmetry of extremal functions. Commun. Pure Appl. Math. 54, 229-258 (2001)

15. Dávila, J. Dupaigne, L: Hardy-type inequalities. J. Eur. Math. Soc 6, 335-365 (2004)

16. Filippas, S, Tertikas, A, Tidblom, J: On the structure of Hardy-Sobolev-Maz'ya inequalities. J. Eur. Math. Soc. 11, 1165-1185 (2009)

doi:10.1186/s13661-014-0232-1

Cite this article as: Monsurrò et al.: Uniqueness results for the Dirichlet problem for higher order elliptic equations in polyhedral angles. Boundary Value Problems 2014 2014:232.

\section{Submit your manuscript to a SpringerOpen ${ }^{\circ}$ journal and benefit from:}

- Convenient online submission

- Rigorous peer review

- Immediate publication on acceptance

Open access: articles freely available online

- High visibility within the field

- Retaining the copyright to your article 